\title{
La ansiedad y la presencia de un desconocido en una clase de lenguas extranjeras
}

\author{
Bryant SMITH ${ }^{\text {a* }}$

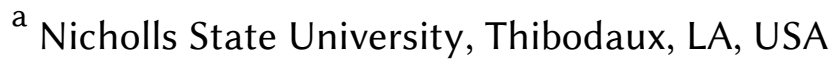 \\ * Corresponding author: james.smith@nicholls.edu
}

\begin{abstract}
A pesar de la abundancia de información sobre el tema de la ansiedad en contextos académicos, las diversas formas en las cuales la ansiedad afecta el rendimiento aún no han sido satisfactoriamente exploradas. Este artículo describe un estudio que se realizó en el cual estudiantes estadounidenses, que tomaban un curso de español en una universidad norteamericana, dieron sus opiniones en varios aspectos relacionados con su ansiedad en el aula de clase. Los resultados indican que los estudiantes que admitieron haber tenido altos y bajos niveles de ansiedad, no tuvieron un buen desempeño en la prueba gramatical en comparación con los que afirmaron sentirse bien. Por otra parte, se evidenció que los involucrados en la prueba no acertaron en el juicio de su propio rendimiento, ya que su punto de vista no coincidió con los resultados de las pruebas aplicadas en este estudio. Por último, y a pesar de la creencia común de que las clases de lenguas extranjeras causan mucha ansiedad, los estudiantes indicaron que otras clases, como ciencias, producen aún más; de esta manera, los resultados indican que en vez de tratar de disminuir la ansiedad en la clase, los maestros deben buscar que los estudiantes se sientan lo más normal que sea posible.
\end{abstract}

Palabras claves:

aprendizaje de idiomas; ansiedad; desempeño en la aula; español.

\section{RESUMEN}

Despite a plethora of information on the topic of anxiety in academic settings, the ways that anxiety affects student performance remain unclear, especially in the foreign language classroom. The present paper describes a study that was conducted in which American students in a university-level Spanish class gave insight into various aspects of their anxiety. Results reveal that students who admit to having both high and low levels of anxiety performed worse on a grammar test than students who admitted to feeling "normal." Students also proved to be poor judges of their own performance as their accuracy was compared to a post-task questionnaire. The presence of a person other than the teacher did not affect the students' performance. Finally, despite its reputation for being anxiety inducing, the students surveyed ranked foreign languages as less stressful than other academic subjects. Results indicate that instead of aiming to remove all anxiety in a foreign language class, instructors might aspire to make students feel as normal as possible.

Keywords:

language learning; anxiety; classroom performance; Spanish.

\section{INTRODUCCIÓN}

\section{La ansiedad}

Todos hemos sentido ansiedad en una situación u otra de la vida. La ansiedad hace parte de la experiencia diaria humana, ya sea en el trabajo o en nuestras relaciones personales. Aunque la existencia de la ansiedad sea inevitable, algo que sí se puede modificar es la reacción que tenemos frente a una situación de estrés. La ansiedad se presenta con más frecuencia en contextos académicos, especialmente cuando los estudiantes se ven enfrentados a procesos de evaluación, sustentaciones, presentaciones, entre otros. En situaciones de tanta presión como éstas, muchos sentimos ansiedad y creemos que puede afectar negativamente nuestro rendimiento en una prueba o examen. Pero en verdad, ¿cuál es el papel de la ansiedad en el aula de clase?

Muchas investigaciones se han realizado sobre la ansiedad que un candidato puede sentir al completar un examen. Muchos investigadores han tratado de estudiar la relación entre el nivel de la ansiedad y el rendimiento de la prueba. Esta correlación, sin embargo, no está tan claramente definida como se podría suponer y muchas de las conclusiones de los investigadores que han estudiado las causas y efectos de la ansiedad en el campo de la educación, son muy variadas.

Varios expertos han llegado a la conclusión de que la ansiedad tiene una influencia negativa en el rendimiento de los estudiantes en la L2 (segunda lengua) en el aula. Supon (2004) confirma los efectos negativos de la ansiedad y propone estrategias para ayudar a los estudiantes a mejorar su rendimiento escolar y bajar su ansiedad (p. 292). Por su parte, Elkhafaifi (2005), en un estudio realizado con hablantes de inglés americano que aprendían árabe, afirma que la ansiedad corresponde negativamente con los logros académicos (p. 206). Por otro lado, Occhipinti (2009) reconoce los efectos negativos de la ansiedad L2, escribiendo "la ansiedad en la clase de lenguas extranjeras es una sensación común que afecta los alumnos en muchas maneras” (p. 81). 
Duxbury y Tsai (2010) insisten que la ansiedad es un fenómeno universal que afecta el rendimiento en las clases de lenguas extranjeras (p. 4). Wang (2010) también señala que la ansiedad es uno de los factores más influénciales en el éxito en su estudio de estudiantes chinos que estudiaban inglés (p. 562). Xu y Li (2010) en un estudio similar confirman los efectos negativos de la ansiedad de los estudiantes en el salón de clase de segunda lengua (L2), explicando como esa ansiedad es un factor negativo que influye en el estado de ánimo de los estudiantes (p. 250). Mahmoodzadeh (2012) aborda las características del alumno que dan pie a la ansiedad en su estudio de estudiantes iraníes que estudiaban inglés (p. 466). Liu (2012), en un estudio con estudiantes taiwaneses de inglés, escribe que más de $80 \%$ de los participantes respondieron que sentían ansiedad y que esta ansiedad afectó su rendimiento en la clase de una manera negativa (p. 132).

Como se señaló anteriormente, hay mucho interés en el tema de la ansiedad a la hora de presentar exámenes. Sin embargo, se necesita más investigación para determinar adecuadamente si hay una posible correlación entre la ansiedad del alumno y los resultados que se obtienen en los exámenes y por otra parte, para entender cómo los estudiantes procesan su ansiedad y cómo se manifiesta en los resultados de sus pruebas. Con la importancia que se les está dando a pruebas estandarizadas, es más urgente que nunca que los educadores entiendan el papel de la ansiedad y el rendimiento de la prueba en la clase de lengua extranjera. Según Supon (2004), "los maestros deben examinar, desarrollar e implementar estrategias para ayudar a los estudiantes a cumplir los logros educativos y poder mejorar su desempeño en las pruebas" (p. 292). Es de esta manera que este estudio intentará hacer que el papel de ansiedad sea entendido mejor y determinar de esta manera, si las precauciones contra la ansiedad son necesarias en el salón de clase o no.

\section{Planteamiento del problema}

Hay mucho interés en el tema de la ansiedad frente a los exámenes. Este tema es especialmente importante hoy en los Estados Unidos debido a legislación impuesta por el gobierno federal. Las nuevas leyes educativas, conocidas como "No Child Left Behind," requieren que las escuelas les den exámenes estandarizados a alumnos entre el tercer y el octavo grado (Supon, 2004, p. 292). Con la nueva importancia que han recibido los resultados de los exámenes (especialmente los exámenes nacionales), muchos educadores se están replanteando el papel que juega la ansiedad en el salón de clase y tienden a dedicar bastante tiempo en disminuirla en sus alumnos. Es así como este estudio espera evidenciar hasta qué grado estas precauciones y estrategias son necesarias.

Es de esta manera que el presente artículo intenta aclarar el papel que juega la ansiedad en el aula clase, ya que si su concepto estuviera mejor definido, los maestros y los alumnos podrían trabajar juntos para mejorar el desempeño en los exámenes. Del mismo modo, en este trabajo se pueden identificar algunas nuevas causas de ansiedad, lo cual podría facilitar el proceso de enseñanza-aprendizaje; de igual manera, puede serle útil al maestro de segundas lenguas y ayudarlo a saber qué es lo que siente el alumno en su clase para que así, este pueda mejorar el uso del idioma.

Finalmente, se desea investigar el poco estudiado efecto de la ansiedad en la clase de segundas lenguas. De las muchas causas posibles de la ansiedad (falta de preparación, falta de sueño, etc.), un factor que ha recibido poca atención, y que aquí toma en cuenta, es la presencia de un desconocido en la clase. Considerando que muchos exámenes son dados por personas que no son los maestros a los cuales los alumnos están acostumbrados (por ejemplo, el SAT y el ACT), los resultados de este estudio podrían ser útiles para los educadores que quieren saber si la persona que administra el examen tiene un efecto en el resultado. Este estudio es especialmente necesario por la controversia que existe sobre el papel de la ansiedad en cuanto al resultado de los exámenes.

Mi deseo es que los hallazgos aquí presentados sirvan para desarrollar el tema de la ansiedad en el salón de clase y en particular, en la clase de lenguas extranjeras. Por otra parte, no solo se intentará responder a la pregunta de si la ansiedad tiene un efecto en el aprendizaje, sino también contestar el siguiente interrogante: ¿Qué relación existe entre la ansiedad y el rendimiento en una prueba?

Anderson y Sauser (1995) expresan lo necesario que es un estudio de este tipo, diciendo: "una mayor comprensión de la relación causa-efecto entre la ansiedad frente a los exámenes y el desempeño en ellos parecería requerir (...) estudios en los que la ansiedad de prueba sirve como una variable dependiente" (p. 20). Ball (1995) indica la necesidad de este estudio, al escribir: "el estado actual de los asuntos relativos a la investigación sobre la ansiedad ante los exámenes indican que (...) los investigadores han sido negligentes al no poner más esfuerzo en el

Smith. B. (2015). La ansiedad y la presencia de un desconocido en una clase de lenguas extranjeras. Latin American Journal of Content and Language Integrated Learning, 8(1), 36-42. doi:10.5294/laclil.2014.8.1.4 elSSN 2322-9721. 
tema (y que) todavía tienen mucho más trabajo por hacer” (p. 113). Esto es justamente lo que se busca realizar en el presente estudio.

\section{La hipótesis}

Para poder controlar nuestras reacciones frente a la ansiedad en la clase, primero hay que identificar algunas de las causas de esta emoción. La pregunta central de este estudio es ¿cómo la presencia de alguien desconocido en el aula de clase afecta el rendimiento de los estudiantes en un examen? Se pensaba que los estudiantes se sentirían más cómodos con el maestro habitual y que sus notas serían más altas. En contraste, en una situación en la cual el maestro regular de los estudiantes no estaba presente, se pensaba que los estudiantes obtendrían peores notas. También se creía que la alta ansiedad afectaría negativamente el rendimiento en un examen y que la baja ansiedad causaría notas más altas. En el presente estudio, se investigará con más detalle el efecto de estas variables..

\section{MÉTODO}

Para llevar a cabo esta investigación y para contestar las anteriores preguntas, se utilizaron dos clases de español intermedio en una universidad pública en los Estados Unidos. Estas dos clases fueron la muestra y había 45 alumnos en total. Cada clase duró 50 minutos aunque la encuesta que llenaron se hizo en 10 minutos. Se escogieron dos clases del mismo nivel de español (nivel intermedio con más o menos el mismo número de estudiantes) para poder comparar los resultados y para que dichos resultados fueran válidos. Es decir, se buscaba un nivel de validez alto para poder confiar en los resultados y poder realizar un segundo proyecto que permitiera generalizar los resultados a la sociedad.

El instrumento central fue un cuestionario que contuvo preguntas cerradas sobre la ansiedad. Es decir, las preguntas eran cerradas y de selección múltiple. Estos cuestionarios son ejemplos de entrevistas estructuradas, porque cada alumno recibió exactamente el mismo instrumento, con el propósito de que la validez interna y externa fueran altas. El hecho de usar cuestionarios en vez de otra manera de conducir investigación hace que el prejuicio del investigador sea menor y que el estímulo sea más estructurado que en una entrevista hecha cara a cara.

El segundo instrumento fue una breve y sencilla prueba gramatical que pretendía medir el rendimiento del alumno. Este "quiz" estaba compuesto por cinco preguntas de selección múltiple sobre un punto gramatical específico. El contenido de esta prueba se basó en el tema que los alumnos estaban aprendiendo el día en que se diligenció el cuestionario. Esta prueba fue el instrumento que midió el rendimiento real del alumno. El cuestionario tenía preguntas que estaban enfocadas a medir las actitudes del estudiante sobre varios aspectos de la ansiedad y su papel en el aula de segunda lengua. Las preguntas sobre la ansiedad estaban relacionadas con las preguntas que espera contestar este estudio. Al usar preguntas cerradas, este estudio fue cuantitativo. Es decir que poder representar los hallazgos de este estudio con cifras, las cuales poder usar en tablas para mejor mostrar los resultados. Este cuestionario fue el instrumento que midió la ansiedad y las actitudes del alumno.

Como se mencionó anteriormente, este estudio se llevó a cabo en dos clases de español (50 minutos) de la misma instructora. En la primera clase, la maestra regular de los alumnos enseñó la clase como siempre y se les dio a sus alumnos una prueba sorpresa. Cuando los alumnos terminaron la prueba, llenaron el cuestionario sobre sus opiniones acerca de la ansiedad. En la segunda clase, la maestra no estuvo presente; en su lugar, el investigador dio la clase y los dos cuestionarios. Al hacer esto, la variable dependiente fue la presencia del investigador, es decir, cómo afecta el rendimiento de una prueba la presencia de un desconocido.

De esta manera, se comparó la diferencia entre el rendimiento de estas dos clases teniendo en cuenta esta variable: la presencia de un desconocido. Sin embargo, este estudio no solo se enfoca en esa variable, sino también en el análisis de cómo la ansiedad afectó el resultado de la prueba, es decir, se pretendía averiguar los resultados obtenidos en la prueba gramatical por los alumnos que admitieron haber sentido mucha ansiedad y viceversa.

Por otra parte, se usó la nota de esta prueba como indicador de éxito y se comparó con las respuestas en cuanto a la ansiedad. Además, se quería establecer si los estudiantes podían predecir cómo actuarían en el examen y si las clases de segunda lengua provocaban más ansiedad que las clases de otro tema, usando las preguntas del cuestionario. Los alumnos indicaron en los cuestionarios su nivel de ansiedad, entonces, ellos mismos fueron

Smith. B. (2015). La ansiedad y la presencia de un desconocido en una clase de lenguas extranjeras. Latin American Journal of Content and Language Integrated Learning, 8(1), 36-42. doi:10.5294/laclil.2014.8.1.4 elSSN 2322-9721. 
quienes decidieron si estaban ansiosos o no. Se compararon a su vez los resultados del cuestionario sobre la ansiedad con el cuestionario gramatical, para ver las relaciones entre los rasgos que este estudio quiere analizar.

Las notas de la prueba gramatical fueron comparadas con las encuestas sobre la ansiedad para poder analizar cómo la ansiedad afectó el rendimiento. Como se vio anteriormente, estos rasgos son parecidos a la pregunta central de esta investigación.

\section{RESULTADOS}

En esta sección se encuentran los resultados de las pruebas que fueron analizados utilizando métodos cuantitativos para analizar las respuestas de los participantes. En cuanto a la pregunta central del estudio (¿qué relación existe entre la ansiedad y el rendimiento en una prueba?), los resultados de la encuesta indican que hay una relación entre estas dos variables, pero que tal conexión no está muy bien definida. Según estos datos, parecería que la hipótesis sobre el papel de la ansiedad en cuanto al rendimiento de un examen fuera correcta. Se ve que los estudiantes con mayor ansiedad tuvieron resultados más bajos que quienes mostraron otro nivel de ansiedad. Algo curioso es que el hecho de estar relajado no subió la nota tanto como el hecho de estar simplemente normal, es decir ni nervioso ni relajado.

Tabla 1. La ansiedad y la nota de la prueba

\begin{tabular}{llllll}
\hline & \multicolumn{2}{l}{ Muy nervioso } & Algo nervioso & Normal & Algo relajado \\
\cline { 2 - 5 } Nota & $50(4)$ & $62.6(19)$ & $65.9(17)$ & $55(4)$ & $60(1)$ \\
\hline
\end{tabular}

La segunda pregunta del estudio (¿la presencia de alguien desconocido en el aula afecta el resultado de un examen?) fue la idea central de este estudio. Se han comparado las notas de las dos clases, una que tenía la maestra habitual y la otra que tenía al investigador (personas desconocidas) como maestro. Estos datos sugieren que la hipótesis de que la presencia de un desconocido bajaría el rendimiento de un examen era correcta. Aunque los estudiantes que hicieron el examen dado por su maestra de siempre sacaron dos puntos y medio más que el otro grupo, esta diferencia no es muy grande.

Tabla 2. El efecto de un desconocido en la clase en la nota.

\begin{tabular}{lll}
\hline & Con la maestra habitual & Con el maestro desconocido \\
\cline { 2 - 3 } Nota & $62.5(25)$ & $60(20)$ \\
\hline
\end{tabular}

Otra pregunta relacionada con un desconocido en la clase es ¿cómo afecta la ansiedad un desconocido en la clase? La sección anterior mostró como el desconocido afecta el rendimiento real de una prueba, mientras que la siguiente muestra como esta persona afecto la ansiedad de los alumnos. Estos datos muestran que no hay mucha diferencia entre la ansiedad de los alumnos en las dos situaciones diferentes. Con el desconocido, $50 \%$ de los estudiantes se sintieron nerviosos, mientras que así se sintieron $52 \%$ de los que hicieron el examen con su maestra de siempre. Aunque la diferencia no es muy grande, los estudiantes estaban más nerviosos con la maestra conocida que con el desconocido.

Tabla 3. El efecto de un desconocido en la clase en la ansiedad

\begin{tabular}{llllll}
\hline & Muy nervioso & Algo nervioso & Normal & Algo relajado & Muy relajado \\
\cline { 2 - 6 } Maestro desconocido & $20 \%(4)$ & $30 \%(6)$ & $40 \%(8)$ & $10 \%(2)$ & $0 \%$ \\
Maestro habitual & $0 \%$ & $52 \%(13)$ & $36 \%(9)$ & $8 \%(2)$ & $4 \%(1)$ \\
\hline
\end{tabular}

La próxima pregunta que se quería contestar con este estudio era: ¿los alumnos son buenos a la hora de predecir su propio rendimiento? Para hacer esto, se compararon las notas de la prueba con la pregunta en el cuestionario que trataba de sus actitudes ante su rendimiento. Estos datos muestran que las mejores notas fueron las de los estudiantes que pensaron que salieron peor que lo normal, mientras que los que pensaron que salieron mejor se equivocaron. Los que juzgaron su rendimiento como "normal" tenían notas significativamente más altas que los que pensaron que salieron "mejor." 
Tabla 4. Los estudiantes como predictores de su propio rendimiento.

\begin{tabular}{llllll}
\hline \multirow{2}{*}{ Nota } & Mucho mejor & Mejor & Normal & Peor & Mucho peor \\
\cline { 2 - 6 } & 0 & 58.5 & 63.4 & 66.1 & 57.5 \\
\hline
\end{tabular}

Para contestar otra pregunta de este estudio (¿generalmente, los alumnos sienten más ansiedad en un aula de segunda lenguas o en una clase de otra materia cualquiera?), se midieron los resultados de un cuestionario. Se ve en esta tabla que la materia que causa más ansiedad es ciencia y no lenguas extranjeras.

Tabla 5. La materia que provoca la más ansiedad.

\begin{tabular}{llllll}
\hline & Matemáticas & Ciencia & Lenguas Extranjeras & Arte/Música & Historia \\
\cline { 2 - 6 } Porcentaje & $34 \%$ & $40 \%$ & $16 \%$ & $5 \%$ & $5 \%$ \\
\hline
\end{tabular}

Después de la ciencia, según los informantes, las matemáticas es la que provoca más ansiedad, mientras que arte, música e historia no parecen causar mucha ansiedad a los alumnos. Otra pregunta formulada durante el proceso de recolección de información fue ¿qué provocó el aumento o descenso de tu nivel de ansiedad?

Tabla 6. Factores que calman la ansiedad.

\begin{tabular}{llllll}
\hline & $\begin{array}{l}\text { El contenido del } \\
\text { examen }\end{array}$ & $\begin{array}{l}\text { La cantidad de } \\
\text { sueño }\end{array}$ & $\begin{array}{l}\text { Persona que da el } \\
\text { examen }\end{array}$ & $\begin{array}{l}\text { El nivel de } \\
\text { preparación }\end{array}$ & Nada \\
\cline { 2 - 6 } Porcentaje & $13 \%$ & $4 \%$ & $2 \%$ & $11 \%$ & $70 \%$ \\
\hline
\end{tabular}

Tabla 7. Factores que aumentaron la ansiedad.

\begin{tabular}{llllll}
\hline & $\begin{array}{l}\text { El contenido del } \\
\text { examen }\end{array}$ & $\begin{array}{l}\text { La cantidad de } \\
\text { sueño }\end{array}$ & $\begin{array}{l}\text { Persona que da el } \\
\text { examen }\end{array}$ & $\begin{array}{l}\text { El nivel de } \\
\text { preparación }\end{array}$ & Nada \\
\cline { 2 - 6 } Porcentaje & $11 \%$ & $7 \%$ & $4 \%$ & $62 \%$ & $16 \%$ \\
\hline
\end{tabular}

El factor que afectó la ansiedad de manera más leve fue la persona que dio el examen. En cuanto al factor que más bajó la ansiedad, la mayoría de los informantes contestaron que nada bajó su ansiedad. Pero el factor que más aumentó la ansiedad fue el nivel de preparación, aunque "nada" constituye la segunda respuesta más común. Las respuestas a la última pregunta son más claras. Por fin, se intentaba saber: ¿qué opinaron los estudiantes sobre la ansiedad en general?

Tabla 8. Las actitudes ante la ansiedad en la clase y su relación con el rendimiento

\begin{tabular}{llllll}
\hline & Baja mucho & Baja poco & No afecta & Sube poco & Sube mucho \\
\cline { 2 - 6 } Porcentaje & $39 \%$ & $44 \%$ & $9 \%$ & $4 \%$ & $4 \%$ \\
\hline
\end{tabular}

Se ve que la gran mayoría (83\%) cree que la ansiedad tiene un efecto negativo en el rendimiento de un examen. Solamente $8 \%$ por ciento de los estudiantes piensan que la ansiedad sube la nota de un examen.

\section{DISCUSIÓN}

Ahora que se ha visto toda la evidencia, hay que intentar una vez más contestar la pregunta: ¿qué papel tiene la ansiedad en la clase? Los resultados han mostrado que esta es una pregunta compleja que tiene muchas facetas. En la primera pregunta, se vio que los estudiantes con mayor ansiedad sacaron las notas más bajas. Esto sugeriría que la ansiedad es la causa y que su presencia hace bajar las notas de una prueba. Pero si esto es verdad, ¿la ausencia de ansiedad no causaría el efecto opuesto? Se evidenció que esto no sucedió. Los estudiantes más relajados no sacaron las mejores notas; al contrario, los mejores estudiantes se sentían simplemente "normal." Puede ser que mucha ansiedad cause efectos negativos, pero lo mejor para un alumno no es excesiva confianza, sino un estado tranquilo o normal.

La idea central de este estudio, el efecto de la presencia de un desconocido en la clase, tal vez causó la reacción más clara de todo el estudio: esta variable no afectó considerablemente la nota de la prueba. Aunque los 
estudiantes que estaban con su maestra de siempre salieron un poco mejor, los resultados no mostraron la gran diferencia que se esperaba. En cuanto al asunto de la diferencia de ansiedad entre los dos grupos, una vez más se ve que no había mucha diferencia. Estos resultados podrían implicar que el hecho de usar una persona desconocida para los alumnos cuando se hacen exámenes no es tan malo.

Según las respuestas a las preguntas sobre las causas de la ansiedad, solo 6\% (el porcentaje más bajo) de los alumnos dijeron que la persona que dio el examen tuvo un efecto en su rendimiento, comparado con $73 \%$ que dijeron que su falta de preparación afectó su nota y $80 \%$ que contestaron "nada." Estos resultados también muestran que la persona que administra el examen no parece tener mucho impacto ni en el alumno ni en su rendimiento. Se ha visto igualmente que los estudiantes no son excelente predictores de su propio rendimiento. Es decir, cuando pensaron que tendrían notas más bajas, la verdad es que salieron mejor que los que pensaron que obtendrían la nota de siempre. También la evidencia de otra pregunta sugiere que las lenguas extranjeras no provocan tanto miedo y ansiedad como las matemáticas o las ciencias.

No es una sorpresa que los estudiantes crean en los afectos negativos de la ansiedad. Aunque no tuvo mucho impacto en su propio rendimiento en la clase, $83 \%$ ciento de los informantes piensa que la ansiedad afecta negativamente su nota. Esta diferencia entre su creencia y la verdad es un poco parecida a la controversia que existe hoy en día en el campo de la enseñanza sobre el papel de la ansiedad. Solamente $8 \%$ de los estudiantes pensaron que la ansiedad podría mejorar su nota, mientras que muchos más educadores piensan lo mismo.

\section{Implicaciones}

Los resultados de este estudio podrían implicar que el énfasis que se pone en la reducción de la ansiedad de los estudiantes es innecesario. Estos datos indican que no hay mucha diferencia entre una persona con mucha ansiedad y una persona muy relajada. Entonces, ¿para qué enfocarse tanto en la ansiedad? Estos resultados, al contrario, parecen indicar que los educadores deberían enfocarse en hacer que los estudiantes se sientan lo más normal posible, en vez de concentrarse en la reducción de la ansiedad. Es decir que los estudiantes no tengan demasiada confianza ni demasiada ansiedad, sino que estén tranquilos y enfocados en la tarea.

\section{Limitaciones}

Una limitación de este estudio fue el tamaño relativamente pequeño de la muestra. Los datos solo reflejan las actitudes de 45 estudiantes. En un futuro estudio, una muestra más grande sería preferible. También, cuando se compararon las dos clases, la clase que tenía la maestra de siempre tenía cinco personas más que la otra. Tal vez hubiera sido más adecuado tener el mismo número de informantes en cada clase.

Otra limitación es que cuando se compara el rendimiento de las dos clases, no se sabe si la nota promedio de los estudiantes era la misma al comienzo de sus estudios. Puede ser que una clase hubiera tenido estudiantes más avanzados que la otra y que esto tal vez haya causado la diferencia de nota, en vez de las otras variables. En un futuro estudio, el investigador debería averiguar si los dos grupos (o clases, en este contexto) tienen un nivel comparable de conocimiento. Este aspecto es especialmente importante en relación con la primera pregunta sobre la ansiedad y el rendimiento.

El cuestionario pide que comparen su rendimiento en la prueba con su rendimiento normal, pero debido a que no se sabe en principio cómo en verdad es su rendimiento, no es un elemento confiable para medir el efecto de la ansiedad en los resultados, de tal manera, hubiera sido mejor preguntar si pensaron que salieron "bien" o "mal," en vez de "mejor" o "peor." Esa sería una sugerencia para un futuro estudio.

\section{Conclusión}

En cuanto al papel de la ansiedad en el aula, los resultados muestran que a pesar de que la ansiedad tiene un efecto en el rendimiento, no tiene un impacto tan negativo como se pensaba anteriormente. Los resultados de este estudio implican que hacer énfasis en la reducción de la ansiedad de los estudiantes es innecesario, ya que los datos indican que hay poca diferencia entre el rendimiento de una persona con una gran cantidad de ansiedad y otra muy relajada. Los factores que afectaron la ansiedad no estaban directamente relacionados con el instructor, sin embargo, elementos como la cantidad de horas de sueño del estudiante o su preparación parecen afectar más significativamente los resultados de las pruebas.

Smith. B. (2015). La ansiedad y la presencia de un desconocido en una clase de lenguas extranjeras. Latin American Journal of Content and Language Integrated Learning, 8(1), 36-42. doi:10.5294/laclil.2014.8.1.4 elSSN 2322-9721. 
Estas deducciones sugieren que los educadores deben concentrarse en hacer que los estudiantes se sienten tan "normal" como sea posible, en lugar de centrarse en la reducción de la ansiedad. Los instructores deben restarle importancia al peso de los "días de prueba" y en su lugar deben tratar de hacer que el ambiente de la clase parezca lo más habitual posible ya que al crear un ambiente en donde los estudiantes se sientan a gusto, los resultados serán más exitosos e enriquecedores.

\section{REFERENCIAS}

Anderson, S.B., and W.I. Sauser. (1995) Measurement of test anxiety: An overview. Philadelphia, PA: Taylor \& Francis.

Ball, S. (1995). Anxiety and test performance. In C. D. Spielberger, and P. R. Vagg (Eds.), Test anxiety: Theory, assessment, and treatment (pp. 107-113). Washington, DC: Taylor \& Francis.

Duxbury, J. G., \& Tsai, L. (2010). The effects of cooperative learning on foreign language anxiety: A comparative study of Taiwanese and American universities. International fournal of Instruction, 3(1), 3-18. Retrieved from http://www.e-iji.net/dosyalar/iji_2010_1_1.pdf

Elkhafaifi, H. (2005). Listening comprehension and anxiety in the Arabic language classroom. The Modern Language fournal, 89(2), 206-220. doi:10.1111/j.1540-4781.2005.00275.x

Liu, H. (2012). Understanding EFL undergraduate anxiety in relation to motivation, autonomy, and language preference. Electronic Journal of Foreign Language Teaching, 9(1), 123-139. Retrieved from http://eflt.nus.edu.sg/v9n12012/liu.pdf

Mahmoodzadeh, M. (2012). Investigating foreign language speaking anxiety within the EFL learner's interlanguage system: The case of Iranian learners. Journal of Language Teaching and Research, 3(3), 466-476. doi:doi:10.4304/jltr.3.3.466-476

Occhipinti, A. (2009). Foreign language anxiety in in-class speaking activities (Unpublished Master's thesis). University of Oslo, Oslo, Norway. Retrieved from https://www.duo.uio.no/bitstream/handle/ 10852/25584/Daxstamparexoggixultima.pdf

Supon, V. (2004). Implementing strategies to assist test-anxious students. Fournal of Instructional Psychology, 31(4), 292-96.

Wang, S. (2010). An experimental study of Chinese English major students' listening anxiety of classroom learning activity at the university level. Journal of Language Teaching and Research, 1(5), 562-568. doi:10.4304/jltr.1.5.562-568.

Xu, R., \& Li, Y. (2010). The effects of teachers' verbal behavior on students' anxiety: Based on the first-year college English classroom in China. Journal of Language Teaching and Research, 1(3), 250-253. doi:10.4304/jltr.1.3.250253.

\section{BIODATA}

Bryant SMITH is an Assistant Professor in the Department of Languages and Literature and Foreign Language Coordinator at Nicholls State University (Thibodaux, LA, USA). He holds a PhD in Linguistics from Louisiana State University (USA). His research interests include Spanish linguistics and comparative and Spanish Golden Age literature. 